\title{
The simulation of news and insiders' influence on stock-market price dynamics in a non-linear model
}

\author{
V. Romanov, O. Naletova, E. Pantileeva \& A. Federyakov \\ Plekhanov Russian Academy of Economics, Russia
}

\begin{abstract}
This work consists of two parts. The first is devoted to stock market simulation and is based on the model described by $\mathrm{Li}$ and Rosser. New factors were introduced in the mentioned model. "Bad" or "good" news arose at each moment. The model has a memory, which determines the rate of forgetting the news. In such a way, the news background is being formed by addition of decaying news intensities. The news background modifies the fundamental value of current market price according to the logistic law. The insiders at moment $\mathrm{t}-1$ know the prices at the moment $t$. The market simulation by means of the proposed model shows that, in case of "good" news, the stock-market prices are rising, and in the case of "bad" news, the prices are falling. Moreover, the parameter that determines the news-forgetting rate changes the picture of rising and falling prices. The model also shows, that the effect of insiders activity depends on the return volume extracted, and when insiders' return approaches some crucial value, the fundamental value $v$ abruptly falls down, and further with the increasing insiders' pressure the market explodes. In the second part of this work, we are considering the possibility for noise-trader to use market states recognition such as "bullish", "bearish", "cyclic" or "stable", instead of using MACD-strategy. With this aim in mind, we applied Kohonen neural net and nonsupervised learning chart pattern recognition.

Keywords: market analysis, news influence, stock market, neural nets, market dynamics, simulation, insiders' role.
\end{abstract}




\section{Introduction}

In addition to the two types of traders in the asset market, described in [1]. F-traders, who could be referred to as "fundamentalists" or "information traders", and $N$-traders, in this article we are considering a small part of market participant, named "insiders". We also take into account another important factor determining the dynamics of the market: the news background. There exist several projects [2-3], the main idea of which is the assumption that news articles might give much better predictions of the stock market than analysis of price history. Thus, authors made an attempt to develop an NLP-system, which takes past news articles and stock prices data as inputs, and builds correlation links between certain features found in these articles and changes in stock prices. The new papers are classified on their influence on the stock market as "plunge" and "surge" or "bad" and "good" news. In Financial Information Grid project the "sentiment" concept is used, that is a qualitative, largely intuitive factor that may influence on the market agent decision, causing either bullish or bearish behavior. The results of this research nevertheless do not permit to analyze in dynamics cumulative interfering and impact of different factors, such as news strength, system memory, period of news accumulation and influence, and market agents strategies. Consequently, the simulation of stock market processes by mean nonlinear discrete models on computers presents powerful alternative way for studying the market, especially in proximity of bifurcation states boarder. In our paper we try to estimate factors that control asset prices in their interaction.

\section{Implementing the news background in the model}

The news influencing estimation is regarded as part of fundamental analysis. Fundamental analysis involves the in-depth analysis of a company's performance and profitability to determine its share price. As new information is released pertaining to the company's status, the expected return on the company's shares changes, affecting the stock price. The advantages of news analysis are its ability to predict changes before they show up on the charts [2-3]. Unfortunately, there is no models formalizing all this knowledge for purposes of automated decision making, and interpretation of news analysis results may be subjective at the high level. The most valuable feature of news analysis is that it makes possible to predict non- stationary points, as rising trend or falling trend, connected with time points, when essential news emerge.

To capture the significant contribution of news analysis to the decision making, we added the news background to our model, thus expanding the system described in article [1]. It replaced or added to the "market noise system". For each day, $k$ random news events $\xi_{k}$ are generated, whose value (or intensity) depends on the position of the "good-bad" slider, and the news sign ("bad" or "good") depends on the slider displacement from the neutral position. The news in this model has a property of being accumulated in time, but the strength of news is decaying with time. The accumulated news comprise the news 
background, which may neutral, positive, or negative, depending on the sign of accumulated news. The weight of news emerged at the moment $k$, in background sum at the moment $j$ is proportional to

$$
\operatorname{Exp}(-u *(j-k)
$$

where parameter $u$ defines the rate of news decay. Then, the total news influence is calculated, based on the news data of all previous days being summed with their weights:

$$
Q_{j}=\sum_{k=0}^{j} \xi_{k} \operatorname{Exp}\left(-u^{*}(j-k)\right.
$$

where $\xi_{k}$ - intensity of news. Then the news background, computed in such way, is applied to modify the price fundamental value for the next day in form of logistic law:

$$
v_{j+1}:=v_{j}+h *\left(\operatorname{Exp} Q_{j}-1\right) /\left(\operatorname{Exp} Q_{j}+1\right),
$$

where $h$ is a constant defining the degree of the news background influence.

The noise factor reintroduced again later. Gaussian white noise with zero mean is generated, and the noise mean value is set by mean program interface window "noise". As a result of the two factors influencing the price fundamental value, the changes in the stock market variable $v$ may be regarded as random walk controlled by news background and noise:

$$
v_{j+1}:=v_{j}+\left(h *\left(\operatorname{Exp} Q_{j}-1\right) /\left(\operatorname{Exp} Q_{j}+1\right)\right)+\varepsilon_{j},
$$

Primarily, in building our model, we have been trying another system, in which the price fundamental value was affected noise as:

$$
v_{i+1}=v_{i}+h^{*} Q_{i} \text {. }
$$

Though it showed similar results, it seemed to us that it was not economically adequate as long as traders rely on the news, and not on "noise".

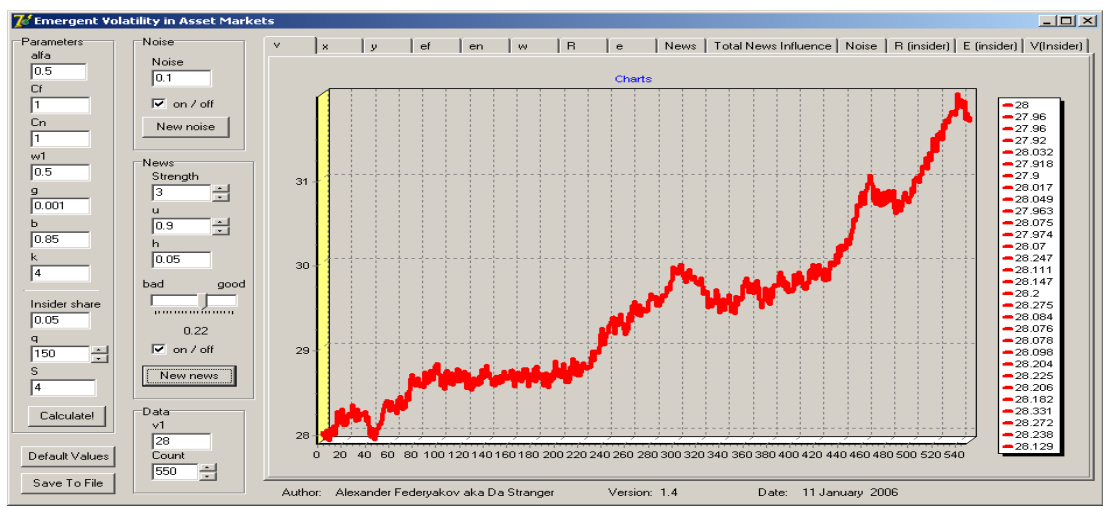

Figure 1: Artificial price fundamental value rising with the influence of good news.

The simulation of the effect of "bad" and "good" news on the market volatility in the model, programmed on Delphi 7.0, showed that rising and falling prices take place in cases of "good" and "bad" news appearing on the market. 
Moreover, the range of price changes depends on the nonnegative parameter $b$, which determines the market sensitivity to the aggregate excess demand $e_{t}$. Figure 1shows the typical picture of artificial course rising with the influence of good news. The lower the rate of news forgetting (long memory), the longer the range of price rising or falling, and the greater the difference between prices at start and end points of the range.

\section{The insiders' model}

The insiders are illegal or semi-legal participants of stock market who get information, which may have essential influence on stock market prices, before this information is officially published in mass media and, as a rule, against the existing laws that forbid its open circulation in the society. The term "insiders" reflects the fact that the specific information they possessed until the current moment was for internal use only inside a limited circle of users. As a result, these persons, getting the insiders information before its official publication, have an opportunity to make the certain moves at the stock market for obtaining superior profits, and thus destabilize the market. The "insiders" parameter was implemented into the model in the following way. Insiders have a certain share of the market in their hands (we designed it " $l$ "). They always receive profit, the value of which can be calculated like this:

eIns $_{t}:=q *\left(x_{t}-x_{t-1}\right)^{2}, q$ is a constant;

the insiders' past relative return of the two trade strategies during the past $k$ periods is estimated as:

$$
\text { Rins }=\left[x_{t} \sum_{j=t-k}^{t-1} e \operatorname{In} s_{j}-\sum_{j=t-k}^{t-1} x_{j} e \operatorname{In} s_{j}\right] / k .
$$

If insiders receive profit, that means someone does not, so we suppose that insiders' past relative return decreases past relative return of other stock market agents:

$$
\begin{aligned}
& \text { IF } R_{t}>0 \quad \text { THEN, } \\
& R_{t+l}=R_{t}-\text { Rins }_{t}, \\
& \text { ELSE } \\
& R_{t+l}:=R_{t}+\text { Rins }_{t},
\end{aligned}
$$

where $R_{t}$ - the past relative return of the two trade strategies during the past $\mathrm{k}$ periods and Rins - insiders' past relative return.

Now, in presence of three types of agents on the market, the aggregate excess demand of the whole market is:

$$
e_{t}=w_{t} * e f_{t}+\left(1-w_{t}-l\right) * e n_{t}+e \operatorname{Ins}_{t} * l .
$$

After that, we calculate the effect that the insiders might have on the price fundamental value. We will describe it here not exactly in all details, but giving a general idea of the process. We have tried 2 variants of insiders influence function on varying fundamental price value.

Variant insiders influence A:

$$
I F\left(R_{t}+\text { Rins }_{t}\right) \neq 0 \text { THEN }
$$




$$
\begin{aligned}
& \operatorname{vins}_{t+1}=v_{t}+\left(h * \operatorname{Exp}\left(-s\left(1-\left(\operatorname{Rins}_{t} /\left(R_{t}+\operatorname{Rins}_{t}\right)\right)\right)\right)\right) *\left(\operatorname{Exp}\left(Q_{j}\right)-1\right) / \\
& \left(\operatorname{Exp}\left(Q_{j}\right)+1\right) \\
& \operatorname{ELSE} \\
& \operatorname{vins}_{t+1}=v_{t}+\left(h *\left(\operatorname{Exp} Q_{t}-1\right) /\left(\operatorname{Exp} Q_{t}+1\right)\right)+\varepsilon_{t} .
\end{aligned}
$$

When we were applying this variant, we could check the combined influence of good news and insiders. Price fundamental value $v$ varying under the influence of good news with the news intensity 0.22 . By the period 540 time units, the artificial rate raises from 28 to 32 . The same picture we can observe with the appearance of bad news, but with only one difference: the artificial course falls down.

Under the insiders impact, the relative rate of which between traders' community constitutes 0.05 and the multiplier value $q=150$, the common view of the plot remains the same, but the scale of course rising decreases and the maximum value achieved is only 28.07 in comparison with 32 without insiders.

Figure 2 shows the impact of the insiders on the fundamental price value in variant $\mathrm{B}$ insiders influence. It is interesting that the insiders get out the profit from the market in big steps as we can see from Figure 2.

In this case, vins represents the new price fundamental value, which includes the insiders' influence on the market. Using the graphical representation you can compare it with the case, what the price could have been without insiders $\left(v_{t}\right)$. Variant insiders influence B:

$$
\begin{aligned}
& I F\left(R_{t}+\operatorname{Rins}_{t}\right) \neq 0 \text { THEN } \\
& \operatorname{vins}_{t+1}=v_{t}+\left(h * \operatorname{Exp}\left(- \text { S }\left(\operatorname{Rins}_{t} /\left(R_{t}+\operatorname{Rins}_{t}\right)\right)\right)\right) *\left(\operatorname{Exp}\left(Q_{j}\right)-1\right) / \\
& \left(\operatorname{Exp}\left(Q_{j}\right)+1\right) \\
& \operatorname{ELSE} \\
& \operatorname{vins}_{t+1}=v_{t}+\left(h *\left(\operatorname{Exp} Q_{t}-1\right) /\left(\operatorname{Exp} Q_{t}+1\right)\right)+\varepsilon_{t} .
\end{aligned}
$$

In this variant, we explored the insiders' impact on the fundamental price value. In this case, it's interesting to note that, in spite of high level of good news (0.54), the fundamental price value after the little jump from 28 to 28.15 only, rises then very slowly (only to 28.24 at 250 days). The next interesting result we

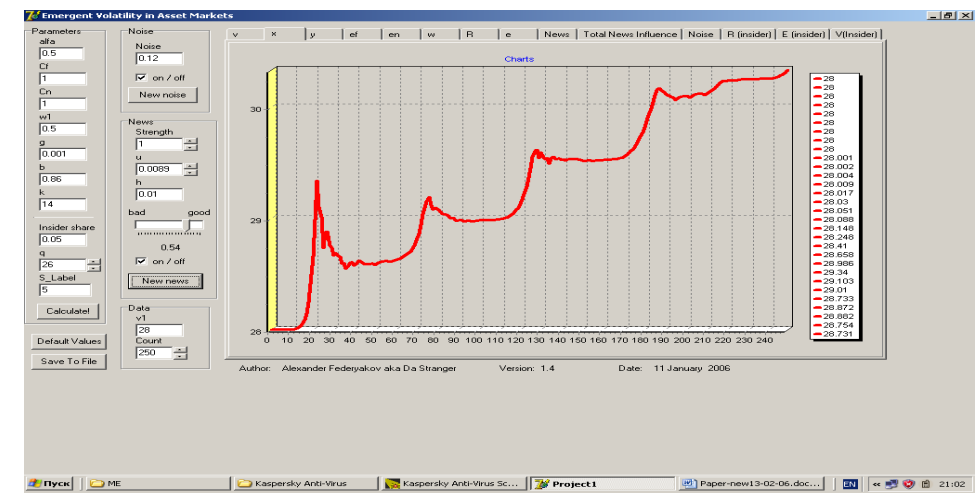

Figure 2: Insiders impact on the assets market price. 
can see in the Figure 2. In spite of practically constant value of the fundamental price, the assets of market prices grow essentially from 28 to more than 30 . Moreover, the plot of prices rising has a stepwise view. This is the consequence of insiders' super-profits (past relative return) which they get from the market. As it is shown in Figure 3, there are the half waves of decreasing heights of the insiders' past relative return strictly at the same moments, when prices plot (Figure 2) makes a jump to the next level.

Probably the time series chart pattern like the one represented in Figure 2 could indicate that the insiders are presenting on the market. It appears that inflation is increasing as insiders do not invest in industry development and rather spent money in consumption sector; and, as a result, the value of fundamental price does not increase while prices at stock market particularly are increasing.

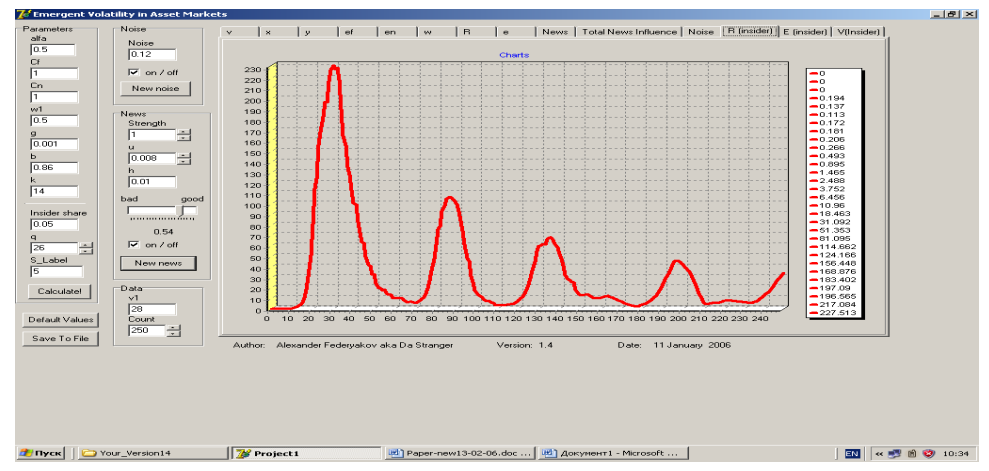

Figure 3: Insiders past relative return.

\section{Kohonen neural net exploring for possible changing N-trader strategy}

In the model described, N-traders are traders of technical analysis or chartist's class, and they are sure that share prices move in trends as a consequence of the constantly changing attitudes of investors in response to different factors. As an alternative to the time series analysis, neural nets could be used. An advantage of neural nets is their capability of learning and adaptation. The neural nets work well in conditions of sharp prices jumps, noisy effects, and contradictory data; thus providing a good addition to the classical technical analysis.

In our case, a data base with variable values (mean weighted buying price, mean weighted selling price, maximum day price, minimum day price, volume) was imported to Excel media from the Central Bank of the Russian Federation site for EURO/ruble and USD/ruble rates for period from 03.10.2003 to 14.10.2005 (about 500 values). The forecast systems use successive values chosen as currency exchange rate:

$$
x(t-n), x(t-n+1), \ldots x(t-1), x(t) \rightarrow x(t+1) .
$$


We assume that there is a certain law that can define the value of the next observation in a time series as function of the past observation values. We suppose also that a finite number $n$ of past observations is enough to determine the next one:

$$
x(t+1)=f(x(t), x(t-1), \ldots x(t-n-1),
$$

The number $\mathrm{n}$ is fixed, and we can neglect the influence of other observations. Having made this assumption, we can speak about a "window" of a size $\mathrm{n}$, which is moving along the time series. The Kohonen neural net must examine the set of values enclosed in the window as a pattern, and try to find pattern classes taxonomy for different window sizes and number of classes specified.

A matter of fact, there is no necessity to slice the input time series to parts according to the window size. It is sufficient to make a column from the input data, take a number of columns that equals the window size, and put these columns together, each of them moved upwards at one unit relatively preceding one. Then each row will represent parts of original time series sliced right at one unit. In this way, we can simulate the window, slicing upon time series and Kohonen net will "see" one after another pieces and try to classify it.

cyclic

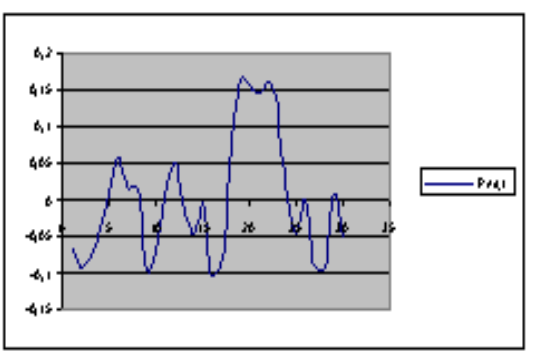

bullish

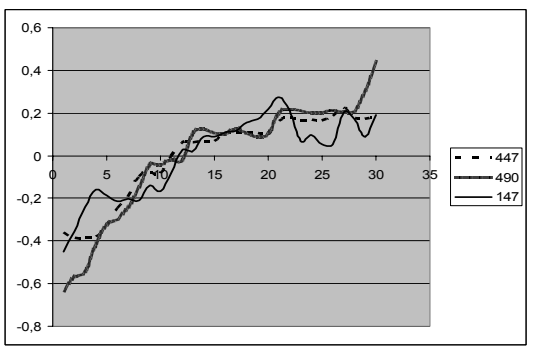

stability

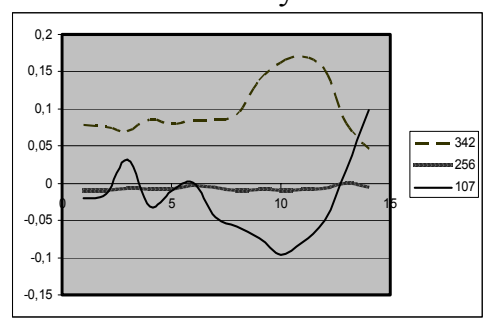

bearish

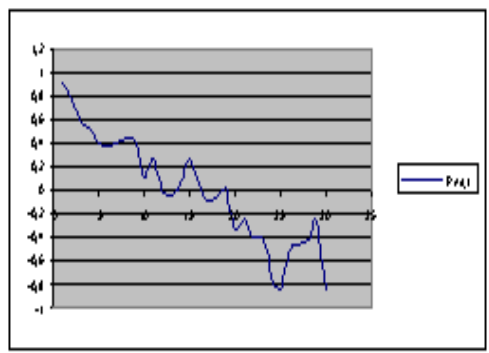

Figure 4: Some pattern classes discovered by Kohonen network.

We use information on different currencies, and its quotations differ from each other very much. We create time series using the original data set (the set of closing prices), and then the average value of time series is calculated. As a 
result, the new time series are obtained after the subtraction of the average from each of the series values. It is possible therefore to avoid the classification based on the level of the prices. Transformed data is imported to ST Neural Networks.

In our work, we have explored $6 \times 6,5 \times 5,4 \times 4$ and $3 \times 3$ Kohonen network, e.g. the network should recognize from 36 to 9 different classes and the window sizes $36,28,21,14$ and 7 days.

In Figure 4, the class discovered by Kohonen network is presented. The class pattern recognition is shown in Figure 5, where the members of the class recognized by the Kohonen net in the time series of EURO/ruble exchange rate are indicated by the arrows.

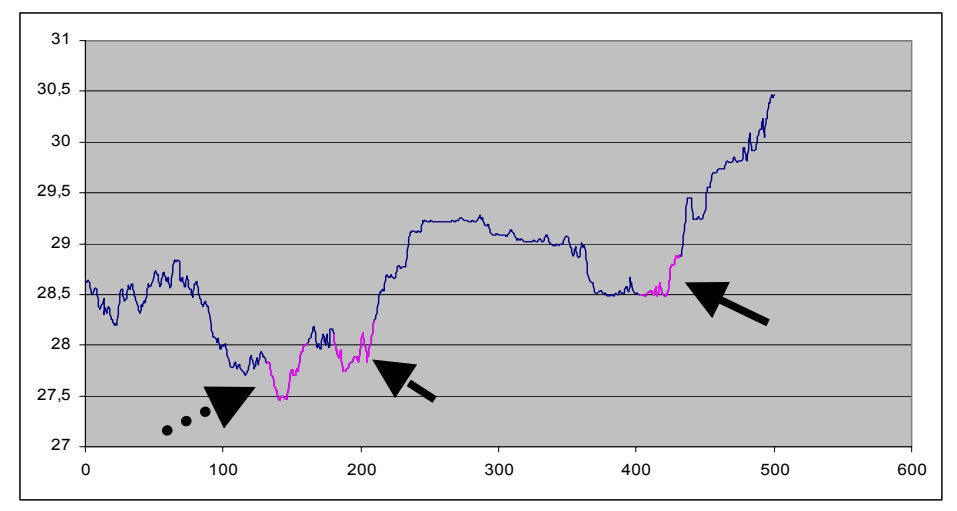

Figure 5: The parts of reversed time series USD/ruble where the arrows indicate that trough was discovered.

The Kohonen networks attempts to find the structure of the data and classes to which data belong. The Kohonen networks can learn to recognize clusters of data, and can also relate chart patterns to each other as well as to clusters. As classes of data are formed, they can be labeled, so that the network achieves capability of classification of new data.

Initially starting with randomly chosen centers of sets, the algorithm gradually adjusts them to reflect the clustering of the training data. The iterative training procedure also arranges the network so that units representing the chart pattern, which are similar in the input space, are situated close together on the topological map. We then say that the KNN has learned. After many epochs, when the KNN has been trained, the weights are frozen, and the test set data are presented to the KNN to examine how well it is able to classify it using the weight values frozen after the training of the KNN.

Our model in some conditions and parameters values has deterministic chaos properties. The R/S-analysis was used for evaluating of persistence features of time series [4]. Our program allows to lead the R/S-analysis of time series and to receive an estimation of Hurst exponent value $\mathrm{H}$.

Figure 6 shows the results of the R/S-analysis and time series for euro at the rate of the Central Bank of the Russian Federation for the period of October 3rd, 2003 through October 14th, 2005. The value of variable $H$ has turned out to be in 
the range of $0,75-0,8$. Calculations were carried out according to the equations published in the article by Skiena [7] As the obtained value of Hurst's exhibitors is concluded in an interval $0,5<\mathrm{h} \leq 1$, it means that the processed time series is persistent and is characterized by the effect of long-term memory.

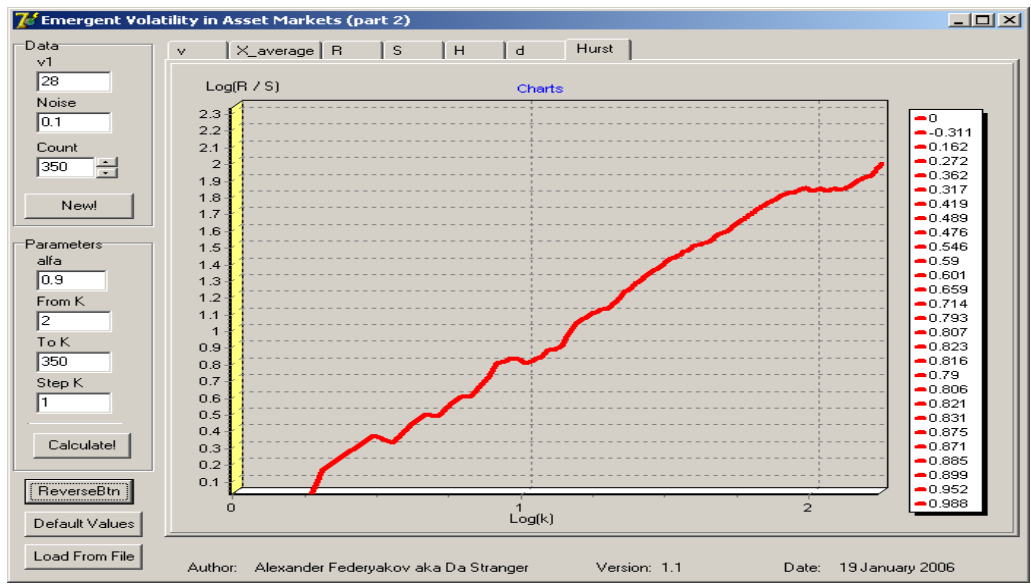

Figure 6: $\quad \mathrm{R} / \mathrm{S}$-analysis for euro at the rate of the Central Bank of the Russian Federation (3.10.2003-14.10.2005).

The learning algorithm uses a time-decaying learning rate, which determines increment of neuron weight adjustment and ensures that the weights alterations become more subtle as the epochs pass. Kohonen net uses topological ordering property, which means that each output neuron has its neighborhood. For getting the best training results, the training process as a rule is conducted in two distinct phases: a relatively short phase with high learning rates and a neighborhood, and a long phase with low learning rate. In our case, the training is conducted in 3 phases, in each phase the number of epochs $(10000 ; 5000 ; 2000)$, the learning rate $(0.1 ; 0.05 ; 0,01)$ and neighborhood $(1 ; 0 ; 0)$ are different. After finishing the network learning, the network may be used for detecting chart pattern in time series.

When training cases were formed, we examine them to see which meaning can be assigned to the clusters in terms of "rise", "fall", "cycle", "stability" and other possible classes. Once the topological map has been built up in this way, new samples from another time series could be submitted to the network for classification.

\section{Conclusions}

The traders' decision must be based on more precise model that we have today, and the future prices forecast must be based on the results of using technical and fundamental analysis. The aim of this article was to research the conditions of traders' activity on the market and to evaluate the effect of different factors. 
The simulation of "bad" and "good" news effect on the market volatility showed that rising and falling of prices takes place in cases "good" and "bad" news appearing on the market. Moreover, the range of price changing depends on the nonnegative parameter $b$, which determines the market sensitivity to the aggregate excess demand $e_{t}$.

The combined influence of good news and insiders in our model shows that, under the impact of insiders, the common profile of rising of the plot course caused by the "good" news remains the same, but the scale of the course rising decreases. It is interesting that the insiders take the profit from the market in big steps.

In one of the model variants, we show that the past insiders' relative return has the profile of periodic peaks corresponding to the stepwise increase in market prices. At the same time, the fundamental price value retains approximately constant.

The experiments with Kohonen network demonstrate that there is a possibility of Kohonen Network learning to recognize the chart pattern specific for time series at hand. The $N$-trader then can make the decision based on the results of program detecting one or another class pattern. The program recognizes the part of time series visible in the "window" as a member of one of the classes that were formed by Kohonen Network.

\section{References}

[1] Honggang Li, J. Barkley Rosser, Jr. Emergent volatility in asset markets with heterogeneous agents. Discrete Dynamics in Nature and Society, vol. 6, no. 3, pp. 171-180, 2001, (www.hindawi.com/journals/ ddns/volume6/S1026022601000188.html)

[2] K. Ahmad, T. Taskaya-Temizel, D. Cheng, L. Gillam, S. Ahmad, H. Traboulsi, J. Nankervis, Financial Information Grid -an ESRC e-Social Science Pilot. Dept. of Computing, University of Surrey, Dept. of Economics, University of Essex.

[3] http://www.ncess.ac.uk/events/past/2004-09-ahm/2004-09-FinGridpaper.pdf

[4] http://www.allhands.org.uk/2004/proceedings/papers/144.pdf, 2004

[5] Hugo van Bunningen, Master of Science Thesis. Augmented Trading: From news articles to stock price predictions using syntactic analysis, University of Twente, (http://www.cyc.com/doc/white papers /Arthur Thesis.pdf, 2004)

[6] Edgar E. Peters, Chaos and Order in the Capital Markets: A New View of Cycles, Prices, and Market Volatility, 2nd Edition, Wiley finance editions, pp. 288, 1996.

[7] Steven S. Skiena "CSE 691 - Lecture 12 - Fractal Analysis" http://www.cs.sunysb.edu/ skiena/691/lectures/lecture12/lecture12.html 